\title{
Primer reporte de Ceroplastes grandis (Hemiptera: Coccidae) en plantas ornamentales de Duranta erecta var. lemon L. (Verbenaceae) en la provincia de Entre Ríos, Argentina
}

Decuyper, C. M., Claps, L. E. y Del Valle, E. E.

DOI: $10.31047 / 1668.298 x . v 37 . n 2.27419$

\section{RESUMEN}

Se realizó un relevamiento de cochinillas blandas pertenecientes al género Ceroplastes Gray, asociadas a Duranta erecta var. Iemon L. en la ciudad de Paraná, Entre Ríos, Argentina. Estos insectos afectan el crecimiento de las plantas, provocando su decaimiento y disminuyendo su valor ornamental. Se realizaron preparaciones microscópicas y se identificó a la especie Ceroplastes grandis Hempel (Hemiptera: Coccomorpha: Coccidae) en la totalidad de las plantas relevadas. Las preparaciones microscópicas fueron depositadas en la Colección de Coccomorpha del Instituto Fundación Miguel Lillo -IFML(Tucumán, Argentina). Se reporta por primera vez en Argentina la asociación de $C$. grandis y plantas de $D$. erecta var. lemon.

Palabras clave: Coccomorpha; cochinillas cerosas; neotropical; planta ornamental.

Decuyper, C. M., Claps, L. E. and Del Valle, E. E., 2020. First record of Ceroplastes grandis Hempel (Hemiptera: Coccidae) in ornamental plants of Duranta erecta var. lemon L. (Verbenaceae) in Entre Ríos Province, Argentina. Agriscientia 37 (2): 53-58

\section{SUMMARY}

The present investigation carried out a survey of soft scales belonging to the genus Ceroplastes Gray associated with Duranta erecta var. lemon L. for ornamental use in Paraná city (Entre Ríos, Argentina). These insects damage the growth of plants, can cause their decay and affect their ornamental value. Through the analysis of microscope slides, the species Ceroplastes grandis Hempel (Hemiptera: Coccomorpha: Coccidae) was identified in all the plants surveyed. The slides were storedin the Coccomorpha Collection Instituto Fundación Miguel Lillo -IFML- (Tucumán, Argentina). The association between C. grandis and plants of $D$. erecta var. lemon is reported for the first time in Argentina.

Key words: Coccomorpha; wax scale; neotropical; ornamental plant. 
Decuyper, C. M. (ORCID: 0000-0003-3075-7752) Facultad de Ciencias Agropecuarias, Universidad Nacional de Entre Ríos, Oro Verde, Argentina. Claps, L. E. (ORCID: 0000-0003-2535-8810): Instituto Superior de Entomología Dr. Abraham Willink (INSUE), Facultad de Ciencias Naturales e Instituto Miguel Lillo, Universidad Nacional de Tucumán, San Miguel de Tucumán, Argentina. Del Valle, E. E. (ORCID: 0000-0003-3972-1721): ICiagro Litoral, Universidad Nacional del Litoral, CONICET, Facultad de Ciencias Agrarias. Correspondencia a: edelvalle@fca.unl.edu.ar

\section{INTRODUCCIÓN}

Duranta erecta var. lemon $\mathrm{L}$. es un arbusto perteneciente a la familia Verbenaceae de utilización ornamental (Parodi y Dimitri, 2004). Debido a su atractivo follaje verde-amarillento, el cual genera un contraste visual deseable con otras especies ornamentales, se las puede observar en los espacios verdes públicos y privados de la ciudad de Paraná, provincia de Entre Ríos, Argentina.

Entre las plagas insectiles que afectan a esta especie vegetal se destacan las cochinillas (Hemiptera: Coccomorpha), que se alimentan de la savia de las plantas y excretan un líquido azucarado llamado melado (Granara de Willink, 2014). Usualmente se encuentran en hojas, tallos o raíces y producen daños en la planta hospedera por la propia succión y por efectos tóxicos de la saliva que inyectan, provocando clorosis y deformaciones en el crecimiento (Kaydan, Kozar y Hodgson, 2015).

Una de las familias más importantes de Coccomorpha es Coccidae, con 177 géneros y 1283 especies (García Morales, Denno, Miller, Miller, Ben-Dov y Hardy, 2016). Estos insectos son conocidos popularmente como cochinillas cerosas y se diferencian de los demás cóccidos por presentar una espesa capa cerosa que recubre el cuerpo de las hembras adultas. La cera se presenta normalmente dividida en placas que se desarrollan alrededor de núcleos centrales que varían, de acuerdo a la especie, en la forma, consistencia, color y tamaño (Peronti, Sousa-Silva y Granara de Willink, 2008).

En Argentina, Granara de Willink y Claps (2003) reportaron cuatro especies del género Ceroplastes Gray asociadas a plantas ornamentales: $C$. cirripediformis Comstock, C. formicarius Hempel, C. grandis Hempel y C. subrotundus Leonardi.
Entre las plantas se mencionan Acacia sp. Mill, Bauhinia sp. L. (Fabaceae), Citrus aurantium L., Citrus sinensis L., Citrus reticulata Blanco (Rutaceae), Fuchsia sp. L. (Onagraceae), Gardenia augusta Ellis (Rubiaceae), Hexachlamys edulis O. Berg (Myrtaceae), Ilex aquifolium L., Ilex paraguariensis St. Hil (Aquifoliaceae), Jacaranda sp. Juss (Bignoniaceae), Phytolacca dioica L. (Phytolaccaceae), Tabebuia lapacho K. Schum., Handroanthus impetiginosus Mattos (Bignoniaceae), Tamarix sp. L. (Tamaricaceae), Schinus molle L. (Anacardiaceae) y Scutia buxifolia Reissek (Rhamnaceae).

En plantas ornamentales de Argentina, la especie C. grandis es la más frecuente y abundante del género. Los adultos se establecen principalmente en ramas jóvenes y las ninfas sobre el tejido foliar, lo que provoca la muerte de ramas y hojas. Además, estos insectos producen abundante melado que disminuye la actividad fotosintética y sirve de sustrato a hongos que se desarrollan sobre las hojas (Granara de Willink y Claps, 2003). Ceroplastes grandis es considerada plaga cuarentenaria en Estados Unidos y el riesgo de invasión es a través de la importación de limones desde Argentina (USDA, 2015).

En Argentina, C. grandis se encuentra en las provincias de Salta, Chaco, Misiones, Tucumán, La Rioja, San Juan, Entre Ríos, Mendoza y Buenos Aires (Granara de Willink, 1999; Granara de Willink y Claps, 2003) y en Brasil Peronti et al. (2008) citan la presencia de $C$. cirripediformis y $C$. grandis sobre Duranta repens var. aurea (Verbenaceae).

El objetivo de la presente investigación fue identificar las especies de Ceroplastes que se encuentran sobre $D$. erecta var. lemon en la ciudad de Paraná (Entre Ríos, Argentina) y reportar su asociación. 


\section{MATERIALES Y MÉTODOS}

\section{Área de estudio y método de muestreo}

El relevamiento se realizó en julio de 2018 en la zona urbana de la ciudad de Paraná ( $31^{\circ} 44^{\prime} 40^{\prime \prime} \mathrm{S}$, $\left.60^{\circ} 31^{\prime} 30^{\prime \prime} \mathrm{O}\right)$; que se encuentra emplazada sobre el margen este del río Paraná a 77 m s.n.m. Se muestrearon plantas de $D$. erecta ubicadas en las veredas de las viviendas y que estaban afectadas por Ceroplastes sp. (Figura 1). Se establecieron 15 sitios de muestreo en diferentes barrios de la ciudad (Figura 2).

En cada sitio de muestreo se recolectaron, mediante tijera de podar, cuatro ramas de $D$. erecta con numerosos individuos de Ceroplastes $\mathrm{sp}$. incluyendo ninfas de los diferentes estadios, hembras maduras y hembras oviplenas. Las ramas fueron colocadas en bolsas plásticas, llevadas al laboratorio y posteriormente los insectos fueron separados manualmente. Se seleccionaron al menos 10 hembras adultas maduras pequeñas (1,5 - 2,0 mm) que no alcanzaron el tamaño final debido a que, cuando llegan a éste, se deforman por estar oviplenas y demasiado juntas, lo que dificulta la tarea de clarificación y montaje.

\section{Montaje e identificación de insectos}

La identificación de Coccomorpha se realizó sobre la base de hembras adultas, mediante preparaciones microscópicas. Para la elaboración de los preparados se siguieron las técnicas de clarificación, deshidratación y montaje permanente

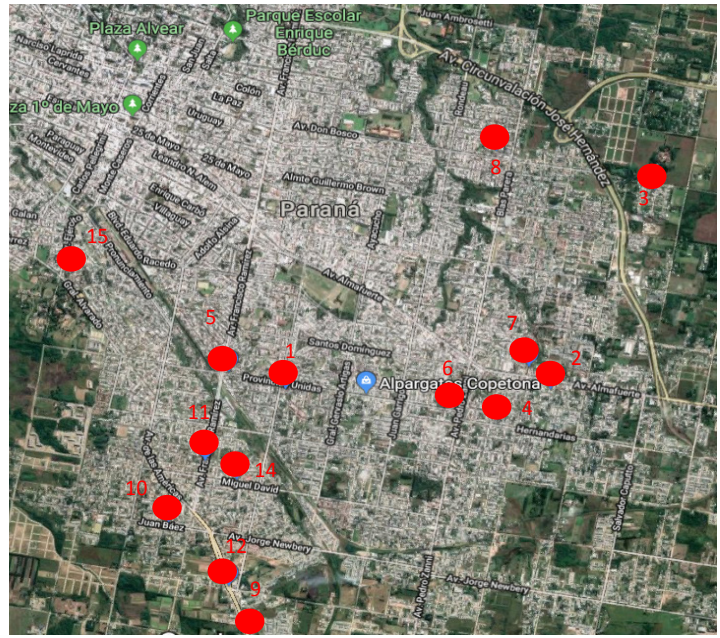

Figura 2. Sitios de muestreo en la ciudad de Paraná. Los puntos rojos señalan los lugares donde se realizaron los muestreos y la numeración se corresponde con la Tabla 1

de acuerdo a Williams y Granara de Willink (1992). Para cada sitio de muestreo se realizó un número variable de preparados microscópicos permanentes que permitieron observar y fotografiar las superficies dorsal y ventral de los insectos (Tabla 1).

La identificación de los ejemplares preparados se realizó utilizando un microscopio óptico Olympus BX51 con contraste de fase y cámara fotográfica incorporada, usando las claves dicotómicas y descripciones de Granara de Willink (1999) y Peronti et al. (2008). El material identificado fue depositado en la Colección de Coccomorpha del

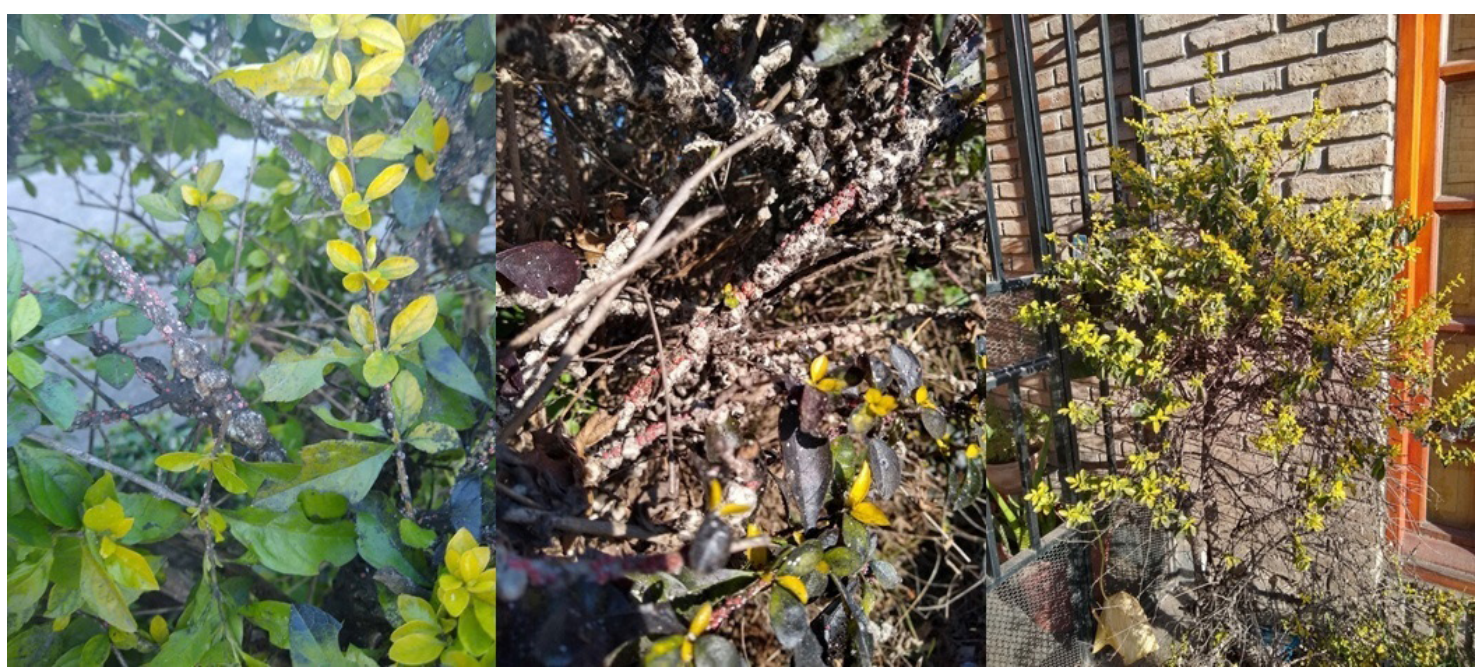

Figura 1. Plantas de D. erecta var. lemon (Verbenaceae) asociadas con poblaciones de C. grandis (Hemiptera: Coccidae) 
Tabla 1. Localización geográfica en la ciudad de Paraná (Entre Ríos) con georreferencia, número de Colección IFML y cantidad de ejemplares hembras de Ceroplastes grandis por preparación microscópica

\begin{tabular}{|c|c|c|c|}
\hline Número & Sitio de muestreo & $\begin{array}{l}\text { Número de } \\
\text { colección } \\
\text { IFML }\end{array}$ & $\begin{array}{l}\text { Cantidad de ejemplares } \\
\text { de hembras adultas por } \\
\text { preparado }\end{array}$ \\
\hline 1 & 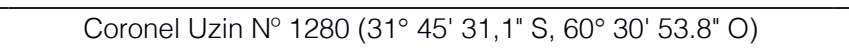 & 1339 & 3 \\
\hline 2 & Gobernador Mansilla № 2250 ( $31^{\circ} 45^{\prime} 31,1^{\prime \prime}$ S, 60²9' 21, 1" O) & 1340 & 3 \\
\hline 3 & Calle $1669 \mathrm{~N}^{\circ} 1503\left(31^{\circ} 44^{\prime} 23.7^{\prime \prime} \mathrm{S}, 60^{\circ} 28^{\prime} 44,8^{\prime \prime} \mathrm{O}\right)$ & 1341 & 1 \\
\hline 4 & Hernandarias $N^{\circ} 2021$ ( $\left.31^{\circ} 45^{\prime} 41,2^{\prime \prime} \mathrm{S}, 60^{\circ} 29^{\prime} 39,7^{\prime \prime} \mathrm{O}\right)$ & 1342 & 1 \\
\hline 5 & Jose Belbei № 26 PA (31 45' 25,3" S, $60^{\circ} 31^{\prime} 13,6^{\prime \prime}$ O) & 1343 & 3 \\
\hline 6 & 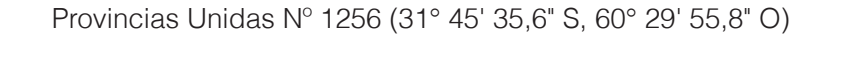 & $\begin{array}{l}1349 \\
1344\end{array}$ & $\begin{array}{l}1 \\
2\end{array}$ \\
\hline 7 & Gobernador Crespo № 30 (31 45' 23,6" S, 60²9' 28,6" O) & $\begin{array}{l}1345 \\
1346\end{array}$ & $\begin{array}{l}2 \\
3\end{array}$ \\
\hline 8 & Don Bosco No 1579 (31 44' 10,6" S, 60²9' 39,9" O) & 1350 & 1 \\
\hline 9 & Avenida de las Américas № 4515 ( $31^{\circ} 46^{\prime} 53,3^{\prime \prime} \mathrm{S}, 60^{\circ} 31^{\prime}$ 05,4" O) & 1351 & 4 \\
\hline 10 & Juan Báez № 179 (31ํ 46' 16,0" S, 60³1' 34,5" O) & $\begin{array}{l}1347 \\
1348\end{array}$ & $\begin{array}{l}3 \\
3\end{array}$ \\
\hline 11 & 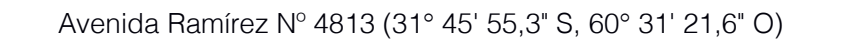 & 1352 & 4 \\
\hline 12 & Avenida de las Américas № 4202 ( $31^{\circ} 46^{\prime} 37,4^{\prime \prime} \mathrm{S}, 60^{\circ} 31^{\prime} 13,5^{\prime \prime}$ O) & 1353 & 4 \\
\hline 13 & De la Torre y Vera $1232\left(31^{\circ} 43^{\prime} 16,2^{\prime \prime} \mathrm{S}, 60^{\circ} 31^{\prime} 15,4^{\prime \prime} \mathrm{O}\right)$ & 1354 & 5 \\
\hline 14 & Miguel David No $254\left(31^{\circ} 46^{\prime} 01,6^{\prime \prime} \mathrm{S}, 60^{\circ} 31^{\prime} 10,7^{\prime \prime} \mathrm{O}\right)$ & 1355 & 3 \\
\hline 15 & Monteagudo $1130\left(31^{\circ} 44^{\prime} 51,1^{\prime \prime} \mathrm{S}, 60^{\circ} 32^{\prime} 08,8^{\prime \prime} \mathrm{O}\right)$ & 1356 & 4 \\
\hline
\end{tabular}

Instituto Fundación Miguel Lillo (IFML) enTucumán, Argentina. Para la identificación de los estadios ninfales se utilizó la clave de Rosa, Peronti, Hodgson y Sousa-Silva (2016).

\section{RESULTADOS Y DISCUSIÓN}

La única especie de cochinilla identificada en el total de los ejemplares analizados $(\mathrm{N}=50)$ (Tabla 1) fue C. grandis. Macroscópicamente se observó en la hembra adulta la cera protectora, blanca con manchas rosadas o completamente rosada; ovalada, circular o irregular en vista dorsal y semicircular o irregular en vista lateral; placas anales no diferenciadas; bandas estigmáticas gruesas. El cuerpo es ovalado, rosa o castaño claro; se oscurece cuanto más viejo es el ejemplar. Los ejemplares presentan una concavidad en la parte ventral que sirve para contener los huevos; llevan un proceso caudal cónico con el ápice dirigido hacia atrás, de color negro. Por su parte, las ninfas se fijan al sustrato y se cubren de sustancias cerosas, de color blanco, con proyecciones laterales. Tienen el aspecto de una estrella y a medida que crecen se cubren totalmente de cera. Los caracteres morfológicos microscópicos de la hembra adulta son los que permiten la identificación de la especie. Éstos se muestran en la Figura 3 y se describen a continuación. Región ventral: antenas con ocho segmentos (Figura 3c), donde se observa que los segmentos III y $\mathrm{V}$ son más largos que el resto, y los segmentos III y IV no poseen setas; un par de pelos interantenales (Figura 3b); patas con digitígulas alargadas en los tarsos, con ensanchamiento apical (Figura 3n); rostro alargado en posición opistognata (Figura $3 \mathrm{~m}$ ); espiráculos con canal estigmático ancho (Figura 3h); setas estigmáticas cónicas (Figura 3k y Figura 3l); setas del margen del cuerpo, con puntas de tamaños diferentes (Figura 3d); placas anales triangulares (Figura 3i); poros decaloculares en la región genital (Figura 3j). Región dorsal: Poro bilocular (Figura 3e), triloculares ovalado (Figura 3f) y triangular y tetralocular (Figura 3g). Las características observadas en el insecto coincidieron con las descripciones de Hempel (1901), Granara de Willink (1999) y Peronti et al. (2008).

Peronti et al. (2008) diferencian a C. grandis de $C$. agrestis Hempel porque la primera posee ocho segmentos en la antena (seis en $C$. agrestis) y también por la presencia de dentículos en la garra (ausentes en $C$. agrestis). Otra especie con la que tiene cercanía es $C$. glomeratus Peronti, que presenta el área esclerosada del proceso caudal con una base amplia que se extiende sobre el dorso, al menos hasta la línea media del cuerpo; mientras que $C$. grandis presenta un área esclerosada del proceso caudal, generalmente circular o alargado, más pequeño dirigido hacia atrás, que no se extiende sobre el área media dorsal.

Se observó que las ninfas caminadoras se 


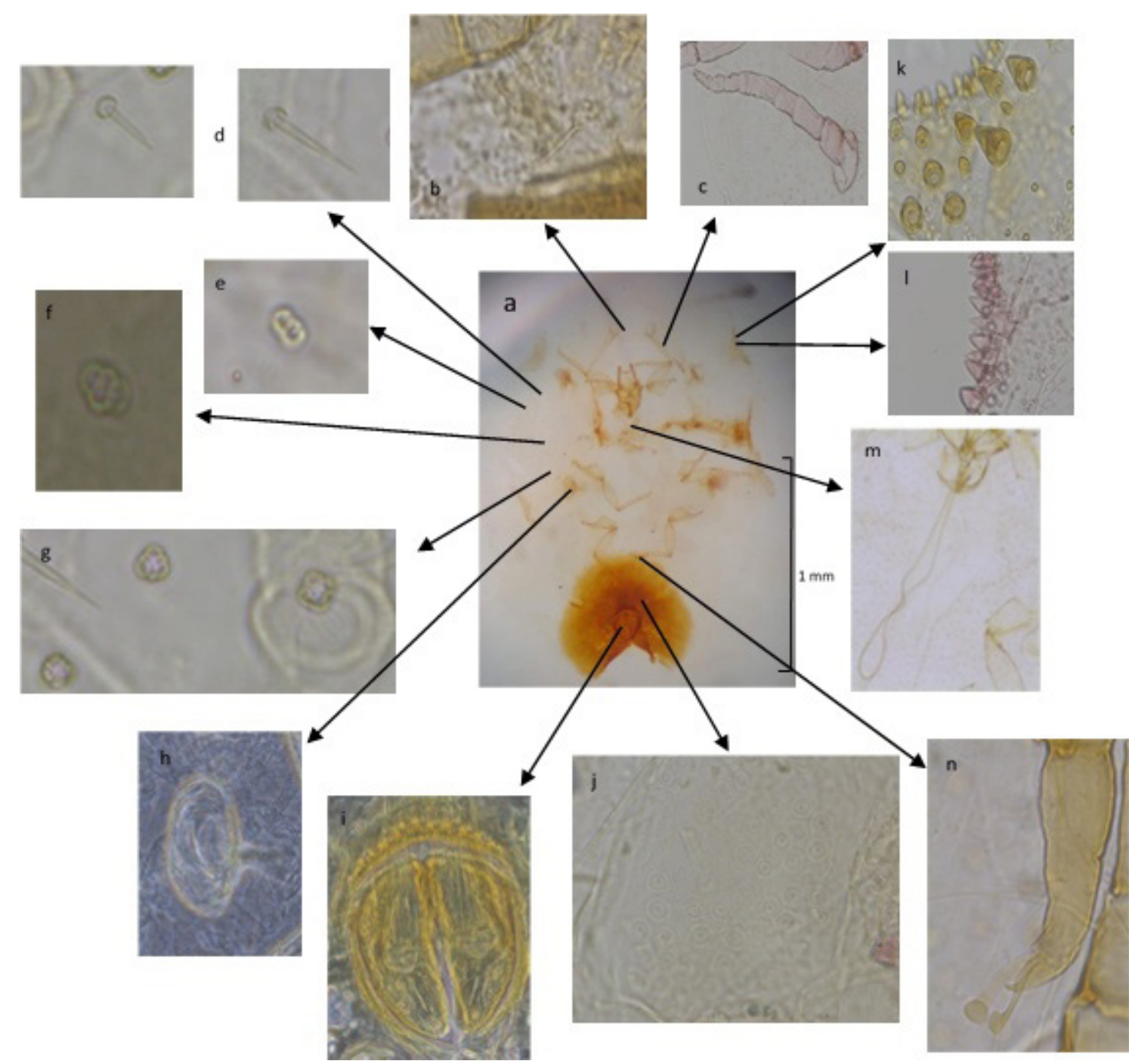

Figura 3. Características morfológicas microscópicas de C. grandis: a) vista ventral del cuerpo completo de hembra adulta de $C$. grandis; b) par de pelos interantenales; c) antena completa; d) setas del margen del cuerpo; e) poro bilocular; f) poro trilocular ovalado; g) poros trilocular triangular y tetralocular; h) espiráculo; i) placas anales; j) poro decalocular de la región genital; k) y l) setas de la región estigmática; m) rostro; n) digitígulas tarsales

desplazan tanto por la superficie foliar como en tallos y ramas, fijándose a sus tejidos, donde pasan el resto de su vida, comienzan a engrosar su capa cerosa (puede alcanzar hasta $1 \mathrm{~cm}$ de espesor) y se fusionan formando masas muy compactas y visibles. En poblaciones altas cubren casi la totalidad del órgano atacado, observándose también los adultos de generaciones anteriores (Figura 1), lo que coincide con lo observado por Correa Franco (2018). Se registra por primera vez en Argentina la asociación entre C. grandis y D. erecta var. lemon.

\section{BIBLIOGRAFÍA}

Correa Franco, L. (2018). Incidencia de la "cochinilla del aguaribay" Ceroplastidia grandis (Hempel) (Hemiptera: Coccidae) en jacarandás (Jacaranda mimosifolia D. Don) del arbolado urbano de la ciudad de La Plata (Buenos Aires, Argentina). Tesis de maestría. Universidad Nacional de La Plata, La Plata, Argentina. DOI: https://doi.org/10.35537/10915/65667

García Morales, M., Denno, B. D., Miller, D. R., Miller, G. L., Ben-Dov, Y. y Hardy, N. B. (2016). ScaleNet: A literature-based model of scale insect biology and 
systematics. Database. DOI: https://doi.org/10.1093/ database/bav118

Granara de Willink, M. C. (1999). Las cochinillas blandas de la República Argentina (Homoptera: Coccoidea: Coccidae). Contributions on Entomology, International, $3(1), 1-183$.

Granara de Willink, M. C. (2014). Hemiptera: Coccidae. En S. Roig-Juñent, L. E. Claps y J. J. Morrone (Eds.), Biodiversidad de Artrópodos Argentinos, Volumen 3 (283-292). San Miguel de Tucumán, Argentina: INSUEUNT Ediciones.

Granara de Willink M. C. y Claps, L. E. (2003). Cochinillas (Hemiptera: Coccoidea) presentes en plantas ornamentales de la Argentina. Neotropical Entomology, 32 (4), 625-637. DOI: http://dx.doi. org/10.1590/S1519-566X2003000400013

Hempel, A. (1901). Descriptions of Brazilian Coccidae. Annals and Magazine of Natural History, 8 (44), 100111. DOI: https://doi.org/10.1080/03745480109442894

Kaydan, M. B., Kozár, F. y Hodgson, C. (2015). A review of the phylogeny of Palaearctic mealybugs (Hemiptera: Coccomorpha: Pseudococcidae). Arthropod Systematics \& Phylogeny, 73, 175-195. Recuperado de: https://www.senckenberg.de/wp-content/uploads/ 2019/08/09_asp_73_1_kaydan_175-195.pdf
Parodi, L. R. y Dimitri, M. L. (2004). Enciclopedia de Agricultura y Jardinería. Tomo 1, Segundo volumen ( $3^{r a}$ ed.). Buenos Aires. Argentina: Acme Agency S.A.C.I y $F$.

Peronti, A. L. B. G., Sousa-Silva, C. R. y Granara de Willink, M. C. (2008). Revisão das espécies de Ceroplastinae Atkinson (Hemiptera, Coccoidea, Coccidae) do Estado de São Paulo, Brasil. Revista Brasileira de Entomologia, 52 (2), 139-181. DOI: http:// dx.doi.org/10.1590/S0085-56262008000200001

Rosa, K. C. C., Peronti, A. L. B. G., Hodgson, C. J. y Sousa-Silva, C. R. (2016). Morphology of the immature female stages and the wax test of the ten species of Ceroplastes (Hemiptera: Coccomorpha: Coccidae: Ceroplastinae) from Brazil. Zootaxa 4136 (2),247-308. DOI: http://doi.org/10.11646/zootaxa.4136.2.2

USDA, United States Departement of Agriculture (2015). Risk Assessment for the Importation of Fresh Lemon (Citrus limon (L.) Burm. f.) Fruit from Northwest Argentina into the Continental United States. (Informe técnico). Raleigh, Carolina del Norte, Estados Unidos: USDA.

Williams, D. J. y Granara de Willink, M. C. (1992). Mealybugs of Central and South America. Wallingford, Reino Unido: CABI Publishing. 